COMMENT. Children diagnosed with benign congenital hypotonia in infancy should be reexamined at intervals through early childhood and should receive extended occupational and other therapy to build muscle strength, balance, and coordination. Hypotonic infants may appear to recover near normal tone by 6 years of age but demonstrate clumsiness and generalized muscle weakness on tests of motor proficiency. My own clinical experience would substantiate these findings. Whereas the office neurological examination appears normal, the mother complains that the child lacks normal stamina, tires easily on extended walks, and often wants to be carried. The symptoms described are sometimes suggestive of a possible myasthenia.

\title{
ACQUIRED NEONATAL BRACHIAL PLEXUS PALSY
}

Three infants presenting at 3, 15, and 21 days of age with brachial-plexus neuropathy were found to have a group-B streptococcal osteomyelitis, as reported from the British Columbia's Children's Hospital, Vancouver, Canada. Osteomyelitis was not recognized initially because the infants were afebrile and generally well. Patient 1 was normal at birth and for the first two days. The parents observed the left arm limp and painful when touched or moved on the 3rd day. An initial diagnosis of traumatic birth injury was changed at 15 days, on examination by neurologists, when the shoulder became warm and swollen, and reflexes were found intact. Blood cultures grew group-B streptococcus and radiographs showed a lytic lesion in the left humerus. EMG and nerve conduction studies on day 28 were consistent with brachial-plexus neuropathy. Intravenous penicillin for 6 weeks was followed by complete recovery by 3 months. Patients 2 and 3 had a similar history and recovered following penicillin therapy. (Sadleir LG, Connolly MB. Acquired brachial-plexus neuropathy in the neonate: a rare presentation of late-onset group-B streptococcal osteomyelitis. Dev Med Child Neurol July 1998;40:496-499). (Respond: Dr Mary B Connolly, Division of Neurology, Department of Paediatrics, British Columbia's Children's Hospital, 4480 Oak St, Vancouver, BC V6H 3V4, Canada).

COMMENT. The authors provide a list of the non-traumatic causes of brachial-plexus neuropathy. In addition to the most common obstetrical injury, a variety of rare, non-traumatic forms are reported, including vaccinations, cytomegalovirus, toxoplasmosis, congenital syphilis, and other infections. Brachial-plexus palsy associated with osteomyelitis may represent a pseudopalsy secondary to pain, or a true paralysis with involvement of the nerves by ischemia or swelling. Nerve conduction studies in the present case reports favor a true paralysis. Early diagnosis and intravenous penicillin therapy result in full recovery. Possible infection in bone should be considered in newborn infants who present soon after a non-traumatic birth with a painful limb paralysis resembling Erb's palsy, despite the absence of fever. For reports of the outcome and diagnosis of obstetric brachial plexus palsy, see Progress in Pediatric Neurology III, PNB Publishers, 1997;pp357-359.

\section{INFLAMMATORY DEMYELINATING POLYRADICULOPATHY}

A clinical and electrodiagnostic, retrospective study of 43 children with acute inflammatory demyelinating polyradiculopathy (AIDP) is reported from the Service de Neuropediatrie, Hopital de Bicetre, France. Age of onset ranged from one to 18 years, less than 3 years in $35 \%$. An antecedent infection occurred within 2 months of onset in $74 \%$. Presenting symptoms included pain (47\%), weakness $(23 \%)$, ataxia $(15 \%)$, and sensory signs $(5 \%)$. When the neurological impairment had ascended and reached a plateau, weakness was noted in $100 \%$, pain in $63 \%$, 
ataxia (23\%), and sensory signs (23\%). Cranial nerve paralyses involved the VIIth in $35 \%$, and extraocular muscles in $16 \%$. Autonomic dysfunction was present in $26 \%$, and $28 \%$ required assisted ventilation. All except 2 recovered completely. Motor and sensory nerve conduction studies showed signs of demyelination during the first week in 90\%, and the diagnosis of AIDP was confirmed in the 2nd week in all patients. A reduced compound muscle action potential was the earliest abnormality (83\%), and motor conduction was abnormal in only $50 \%$, during week 1. The outcome was better in children than in adults. (Delanoe C, Sebire G, Landrieu P, Huault G, Metral S. Acute inflammatory demyelinating polyradiculopathy in children: Clinical and electrodiagnostic studies. Ann Neurol Sept 1998;44:350-356). (Respond: Dr Metral, Service d'Explorations Fonctionnelles du Systeme Nerveux, 78 Rue du General Leclerc, 94275 Kremlin, Bicetre Cedex, France).

COMMENT. Based on electrophysiologic criteria, the diagnosis of AIDP may be suspected in the first week and confirmed in the second week of the illness. The clinical manifestations in the acute phase are similar in children and adults, but the outcome of AIDP in children is better. More than one-third of children affected are under 3 years of age. Severe limb or back pain, weakness, and ataxia are the most prominent initial symptoms.

In contrast to the acute form of inflammatory demyelinating polyneuropathy (IDP), the chronic form presents with lower extremity weakness and difficulty in walking in $85 \%$. The majority have a poor long-term prognosis, with frequent relapses and residual weakness (Progress in Pediatric Neurology III, PNB Publ, 1997;pp360-362).

Correlation between cytomegalovirus infection and IgM antiMAG/SGPG antibody-associated neuropathy (Yuki N, Yamamoto T, Hirata K. Ann Neurol Sept 1998;44:408-410) is reported from Dokkyo University, Tochigi, Japan. CMV infection may induce the IgM anti-myelin-associated glycoprotein antibody found in some patients with chronic polyneuropathy.

\section{ATTENTION DEFICIT AND LEARNING DISORDERS}

\section{EFFECT OF TRAUMATIC BRAIN INJURY ON ADHD SYMPTOMS}

The course of attention-deficit hyperactivity (ADH) symptomatology in 50 children and adolescents after traumatic brain injury (TBI) at 6 to 14 years of age was studied prospectively at the University of Iowa, Iowa City, IA. Changes in the $\mathrm{ADH}$ symptomatology and early onset of hyperactivity in the first 2 years after TBI were significantly related to the severity of the injury and degree of brain damage, and to a measure of family dysfunction, but a correlation with MRI findings was not documented. (Max JE, Arndt S, Castillo CS et al. Attention-deficit hyperactivity symptomatology after traumatic brain injury: a prospective study. I Am Acad Child Adolesc Psychiatry Aug 1998;37:841-847). (Reprints: Dr Jeffrey E Max, Department of Psychiatry, University of Iowa, 1876 JPP, Iowa City, IA 52242).

COMMENT. This study confirms previous reports of the relation between head injury and brain damage and the ADHD syndrome. It also demonstrates a positive "dose-response" relationship between severity of injury and change in $\mathrm{ADH}$ symptoms. It is unfortunate that the MRI findings in this series of patients could not document a neuroanatomical correlate of the post-TBI change in ADH symptoms. The literature regarding head injury and ADHD is reviewed in Attention Deficit Hyperactivity and Learning Disorders, PNB Publ, 1998;pp 14-21. 